\title{
Intensity scale of side effects in European sea bass (Dicentrarchus labrax) post intraperitoneal injection with commercial oil-adjuvanted vaccines
}

\author{
H. Tziouvas, MSc. ${ }^{1}$, P. Varvarigos ${ }^{2}$ \\ ${ }^{1}$ Benchmark Genetics Ltd UK, ${ }^{2}$ Freelance Veterinary Consultant, AquaHealth \\ Keywords: Sea bass, vaccination, side effects, adhesions, adjuvant, intra peritoneal \\ https://doi.org/10.48045/001c.28222
}

Bulletin of the European Association of Fish Pathologists

Vol. 41, Issue 3, 2021

\begin{abstract}
Intraperitoneal (i.p.) injection vaccination is considered effective to protect European sea bass (Dicentrarchus labrax) from economically important diseases. Water-based as well as oil-adjuvanted vaccine formulations are being widely used. The oil adjuvants used to potentiate antigen effects lead to higher and more prolonged immune response but also to chronic peritoneal inflammatory reactions, regarded as adverse or side-effects. During a three-year field study conducted throughout Greek mariculture, the intra-abdominal lesions were assessed on fish sampled prior to, and at different times post i.p. injection with different oil-adjuvanted vaccine formulations. The scope was to create a macroscopic side-effect intensity scale for sea bass in line with those applicable to salmonids (Salmo salar, Oncorbynchus mykiss) and cod (Gadus morbua). The diversity of lesions observed ranged from none (presumably non-injected; score 0 ) to widespread, strong adhesions with granuloma, pigmented or not (score 6). The occurrence of small nodules apparently containing vaccine remains, encapsulated by layers of connective tissue is described. The proposed scoring system is described and illustrated, intended to guide farm staff to evaluate the intensity of post-injection vaccination side-effects by necropsy on site.
\end{abstract}

\section{Introduction}

European sea bass (Dicentrarchus labrax) and Gilthead sea bream (Sparus aurata) are the two most economically important aquaculture species in the Mediterranean. According to the Federation of Greek Maricultures (FGM) 2020 report, sea bream and sea bass 2019 world production from aquaculture was 466,083 metric tons, up 5,2\% over 2018. Sea bream accounted for $54.1 \%$ and sea bass for $45.8 \%$ of total production. These two species account for almost $25 \%$ of volume and $28 \%$ of value of the total European fish culture production. Both species are cultured in eight European Union (EU) Mediterranean countries (from east to west: Cyprus, Greece, Croatia, Italy, Malta, France, Spain, Portugal) with a total of 204,200 metric tons produced in 2019 .

The most important diseases of sea bass are infections caused by the Gram negative bacterium Listonella anguillarum (syn. Vibrio anguillarum -Va), the Gram negative bacterium Photobacterium damselae subspecies piscicida (Phdp) and viral encephalopathy and retinopathy (VER; syn. viral nervous necrosis -VNN) caused by a piscine betanodavirus (NNV) (Vendramin et al. 2016; Muniesa et al. 2020). Vaccination has become an essential part of fish farming practice contemplated as a cost-effective approach to successfully manage infectious diseases that threaten the aquaculture industry (Tafalla, 
Bøgwald, and Dalmo 2013). For effective disease control, a number of vaccines that present the antigens $(\mathrm{Ag})$ in oil adjuvanted formulations are administered by intraperitoneal injection (i.p.). However, a variety of adverse side effects, such as peritoneal adhesions and pigmentation have been studied extensively for salmonids, thus the so-called Speilberg scale was established to describe their intensity (Midtlyng, Reitan, and Speilberg 1996). A similar side-effect scale has been developed for Atlantic cod (Maira, Lystad, and Alexandersen 2007). Although oil-adjuvanted injectable vaccines are widely used in commercial sea bass production, an intensity scale has not yet been developed for this species. Thus, the objective of this study was to create a macroscopic post i.p. injection vaccination side-effect intensity scale specific to sea bass.

\section{Materials and methods}

The work methodology considered the guidelines for the evaluation of the safety and efficacy of fish vaccines in the field (EMA/CVMP/IWP, 2011) with the scope to produce a field scoring guide for intraperitoneal side-effects across oil-adjuvanted vaccine formulations. Field assessment studies were performed on a range of different on-growing cage sites in Greece throughout a three-year period (2016-2018). Each necropsied sample consisted of at least 30 fish from each population/net pen. Each sampled population was examined both preand post- vaccination. Apart from the necropsied fish, the weights of at least 100 additional fish at each sampling point were taken. Sampling was conducted throughout the summer and into late autumn (late June to late October).

The vaccines that were administered i.p. to the fish comprised monovalent (nodavirus nervous necrosis virus; NNV), divalent (Vibrio anguillarum; Va, and Photobacterium damsellae Sub sp. piscicida; Phdp) and trivalent (Va, Phdp, NNV) commercially licensed formulations or experimental formulations approved for field trial assessment. The vaccines were produced by three different manufacturers using either mineral or non-mineral oil-based adjuvants.

The study assessed fish both prior to and post vaccination, from twenty three different cages at fourteen different sites, belonging to five aquaculture companies. Fish at each site and sampling point were captured during early daylight hours prior to first feeding using the sweep net method. Farm staff teams using the sweep nets either employed hand hauling techniques or deployed the net by means of a telescopic crane fixed on a service boat or raft. The captured fish where then placed in 500L tanks and were euthanised by an overdose of licensed anaesthetic. The fish were necropsied on site for biosecurity compliance. The examination for peritoneal lesions was performed by revealing the peritoneal cavity surgically, as shown in Figure 1. Photographic records and descriptive notes of the observed side-effects were taken per individual fish sampled. 


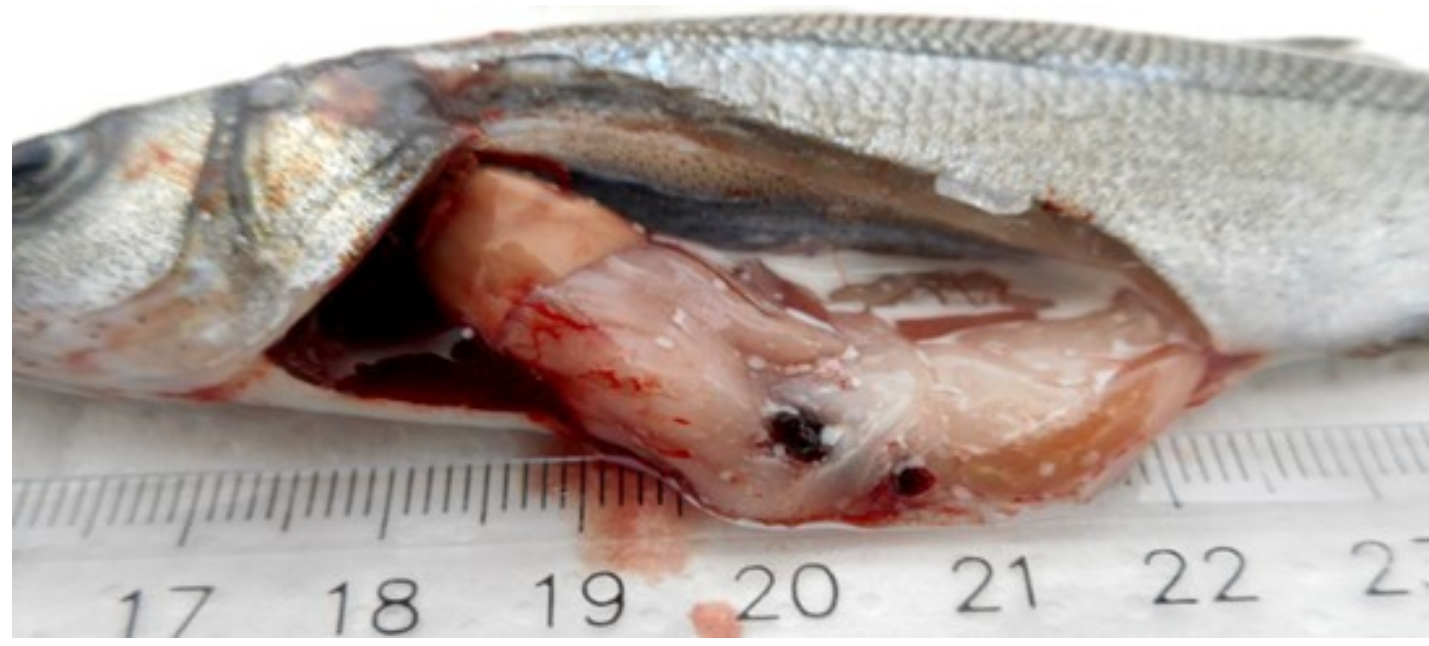

Figure 1. Peritoneal cavity of a sea bass opened immediately post i.p. vaccination. Droplets as well as a mass of oiladjuvanted vaccine are seen.

Different cage populations were sampled from the same site each season, since different populations of sea bass were evaluated each year. No population was sampled twice during final rearing, hence, there is no data with regards to the progress of these lesions throughout harvest.

Table 1 shows details of the sea bass groups sampled at 14 different culture sites in Greece and the vaccination year. Timing of necropsies post vaccination ranged from 13 days up to 161 days (median 67 days), and from 304 DD up to $3542 \mathrm{DD}$ (median $1540 \mathrm{DD}$ ). The identity of each company and site are not disclosed but are given using a simple alphanumeric system. Although histology was not intended to be part of the study, several pieces of peritoneal tissues carrying nodular lesions were fixed in $10 \%$ neutralised buffered formalin $(\mathrm{NBF})$ and haematoxylin-eosin stained tissue slides were prepared.

\section{Results and discussion}

Injection of salmonids with oil-adjuvanted vaccine formulations often provoke a peritoneal reaction/inflammation leading to side-effects of variable intensity (Mutoloki et al. 2006; Villumsen, Koppang, and Raida 2015). In the Mediterranean, injection vaccination is recommended when sea bass are not smaller than 15g. Despite this, occasionally, farmers inject smaller sized fish, even down to $10 \mathrm{~g}$, in order to comply with time plans. Fish are injected either by hand, by means of trained crews using "injection guns", or by injection machines. In sea bass, the recommended injection site is at the abdominal mid-line about $5 \mathrm{~mm}$ posterior to the tip of the pelvic fins as shown in Figure 2. Nevertheless, at times, when staff are in a hurry or the machines are not properly calibrated in accordance with the size of the fish, the recommended injection site is missed and this may lead to internal organ injuries, occasionally associated with bleeding or skin and abdominal muscle tears. If fish are mishandled, then mortalities can result soon after injection. 
Table 1. Description of the populations from which samples were evaluated. Average weight of the populations examined at each site estimated at vaccination (control sample) and post vaccination are based on the sampled fishes +100 non-necropsied individuals.

\begin{tabular}{|c|c|c|c|c|c|c|}
\hline $\begin{array}{l}\text { Company } \\
\text { /Site }\end{array}$ & Year & $\begin{array}{c}\text { Average weight at } \\
\text { vaccination }(\mathrm{g}) \pm 2 \times \mathrm{xD}(\mathrm{n} \\
=100)\end{array}$ & $\begin{array}{l}\text { Days post- } \\
\text { vaccination }\end{array}$ & $\begin{array}{l}\text { Average sea } \\
\text { temperature } \\
\left({ }^{\circ} \mathrm{C}\right)\end{array}$ & $\begin{array}{c}\text { DD post } \\
\text { vaccination }\end{array}$ & $\begin{array}{c}\text { Average weight } \\
\text { post } \\
\text { vaccination }(g) \\
\pm 2 \times S D(n=100)\end{array}$ \\
\hline \multirow{4}{*}{$A / 1$} & 2016 & $35.5( \pm 7.64)$ & 29 & 25 & 725 & $45.4( \pm 9.32)$ \\
\hline & \multirow{3}{*}{2018} & $140.5( \pm 35.3)$ & 44 & 26 & 1144 & $181.20( \pm 38.1)$ \\
\hline & & $180.64( \pm 44.5)$ & 44 & 26 & 1144 & $210.3( \pm 51.9)$ \\
\hline & & $147.6( \pm 25.5)$ & 44 & 26 & 1144 & $175.0( \pm 47.2)$ \\
\hline \multirow{2}{*}{$A / 2$} & \multirow{2}{*}{2017} & $85.12( \pm 43.12)$ & 110 & 25 & 2750 & $133.17( \pm 26.04)$ \\
\hline & & $112.15( \pm 22.14)$ & 110 & 25 & 2750 & $148.43( \pm 31.15)$ \\
\hline \multirow{2}{*}{$B / 1$} & 2016 & $20.68( \pm 2.23)$ & 21 & 22 & 461 & $27.0( \pm 5.15)$ \\
\hline & 2017 & $77.64( \pm 15.74)$ & 103 & 25 & 2574 & $135.23( \pm 22.45)$ \\
\hline $\mathrm{C} / 1$ & 2016 & $44.42( \pm 54.12)$ & 161 & 22 & 3542 & $87.3( \pm 24.84)$ \\
\hline $\mathrm{C} / 2$ & 2016 & $56.42( \pm 34.65)$ & 45 & 25 & 1127 & $74.2( \pm 17.50)$ \\
\hline $\mathrm{C} / 3$ & 2016 & $32.21( \pm 43.78)$ & 101 & 24 & 2426 & $62.5( \pm 11.79)$ \\
\hline $\mathrm{C} / 4$ & 2016 & $32.28( \pm 36.1)$ & 87 & 25 & 2175 & $64.4( \pm 18.12)$ \\
\hline $\mathrm{D} / 1$ & 2016 & $18.92( \pm 15.41)$ & 13 & 23 & 304 & $24.8( \pm 3.14)$ \\
\hline $\mathrm{D} / 2$ & 2017 & $97.23( \pm 74.90)$ & 20 & 24 & 480 & $125.0( \pm 20.12)$ \\
\hline$D / 3$ & 2017 & $87.86( \pm 34.70)$ & 132 & 24 & 3168 & $157.1( \pm 28.90)$ \\
\hline \multirow{2}{*}{$E / 1$} & \multirow{2}{*}{2016} & $57.42( \pm 65.21)$ & 30 & 23 & 690 & $73.4( \pm 12.19)$ \\
\hline & & $25.62( \pm 54.24)$ & 25 & 23 & 567 & $37.2( \pm 7.74)$ \\
\hline $\mathrm{E} / 2$ & 2016 & $44.22( \pm 72.63)$ & 52 & 24 & 1253 & $67.3( \pm 12.36)$ \\
\hline$E / 3$ & 2017 & $77.45( \pm 62.44)$ & 92 & 24 & 2222 & $127.07( \pm 16.30)$ \\
\hline \multirow{4}{*}{$\mathrm{E} / 5$} & \multirow{2}{*}{2017} & $105.63( \pm 21.92)$ & 138 & 22 & 3036 & $178.57( \pm 30.47)$ \\
\hline & & $112.61( \pm 48.22)$ & 102 & 22 & 2244 & $187.7( \pm 32.16)$ \\
\hline & \multirow{2}{*}{2018} & $80.82( \pm 47.21)$ & 67 & 23 & 1540 & $109.6( \pm 28.9)$ \\
\hline & & $73.63( \pm 73.56)$ & 80 & 23 & 1848 & $110.2( \pm 27.0)$ \\
\hline
\end{tabular}

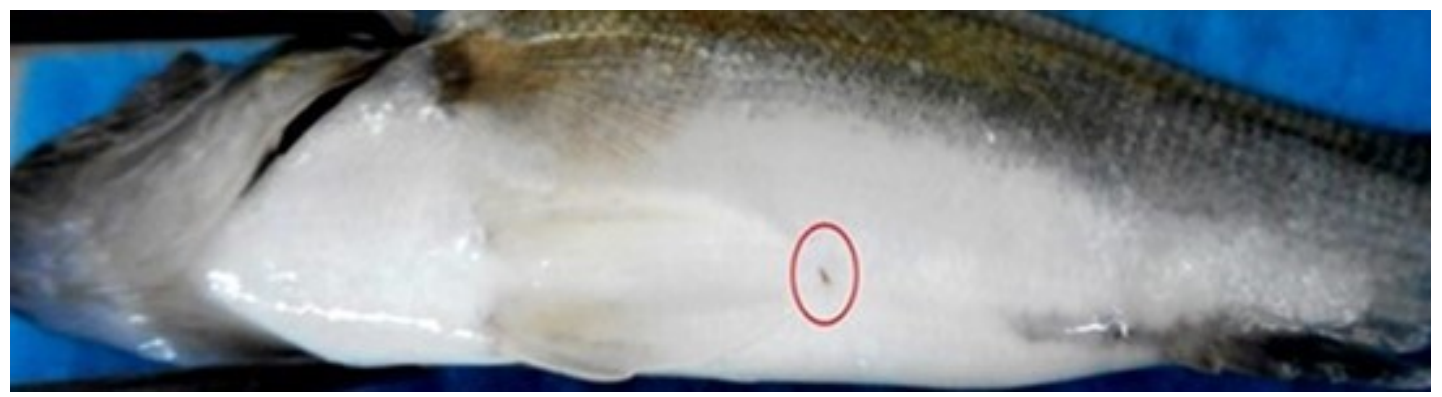

Figure 2. Recommended injection site for European sea bass.

Side-effects due to a peritoneal inflammatory reaction against oil adjuvanted vaccines raised concerns regarding their safety; the observed side effects included intra-abdominal peritoneal lesions due to chronic peritonitis, such as adhesions of visceral peritoneum hymens between the organs and the parietal 
peritoneum hymens lining the abdominal wall, peritoneal granulomatous lesions (nodules) and pigmentation (Mutoloki et al. 2006; Villumsen, Koppang, and Raida 2015).

A comparative histological study of the peritoneal lesions in sea bass subsequent to i.p. administration with different vaccine formulations was conducted by Afonso et al. (2005) who attempted to associate the intensity of the intraperitoneal lesions with the observed histopathology by means of a laboratory scale experiment. Short and long term histopathological effects one, two, four and eleven months after i.p. injections with different bacterial vaccine formulations, monovalent (Va) or divalent (Va plus Phdp), were studied as well as the effects of different vaccine adjuvants, mineral oil, liposome or alginate. In contrast to that histological study, the present work focused on necropsy and the macroscopic characteristics of intra-abdominal lesions in the field.

Nodular structures comprise large granulomatous lesions encapsulating the vaccine, acting as depots. When these granulomatous lesions burst by pressure or are cut open, the oily vaccine content within is revealed. In order to confirm the structure of such lesions, we chose to perform occasional microscopy of fresh nodular peritoneal hymens and histology of fixed nodular tissue. Under relatively low magnification, microscopy of fresh tissue revealed reactions of the peritoneal connective tissue and encapsulation of the vaccine as shown in Figure 3. Indicative microscopy fields from HE stained slides are shown in Figure 4 , revealing distinct connective tissue layers around the vaccine droplets and moderate leucocyte infiltration of the tissue surrounding the nodules. No melanomacrophages were noted. The findings were in accordance with the study by Afonso et al. (2005).

Abdominal side-effects following vaccination may undermine fish welfare, but a field applicable severity scale is so far lacking for sea bass, despite the extensive application of oil-adjuvanted i.p. injectable vaccines in commercial sea bass farming.

Despite the observation of visible side-effects in the visceral cavity, there were no visible lesions left on the carcass after evisceration and no observations nor ex farm reports have associated the observed adverse reactions with negative effects on growth or feed conversion ratio (FCR), except for cases where the fish suffered widespread extreme score 6 side-effects with retarded growth and muscle emaciation, potentially due to obstruction of the normal intestinal movements.

Although it was not the intention to associate fish size and vaccine type with the peritoneal lesions intensity, it became obvious during the course of the study that the smaller the fish when vaccinated the more pronounced the observed side-effects. This is in agreement with findings in salmon (Berg et al. 2006). Free residues of the oil-based vaccines were not visible and all vaccine droplets seemed processed or enclosed in nodules at $240 \mathrm{DD}$ or about 10 


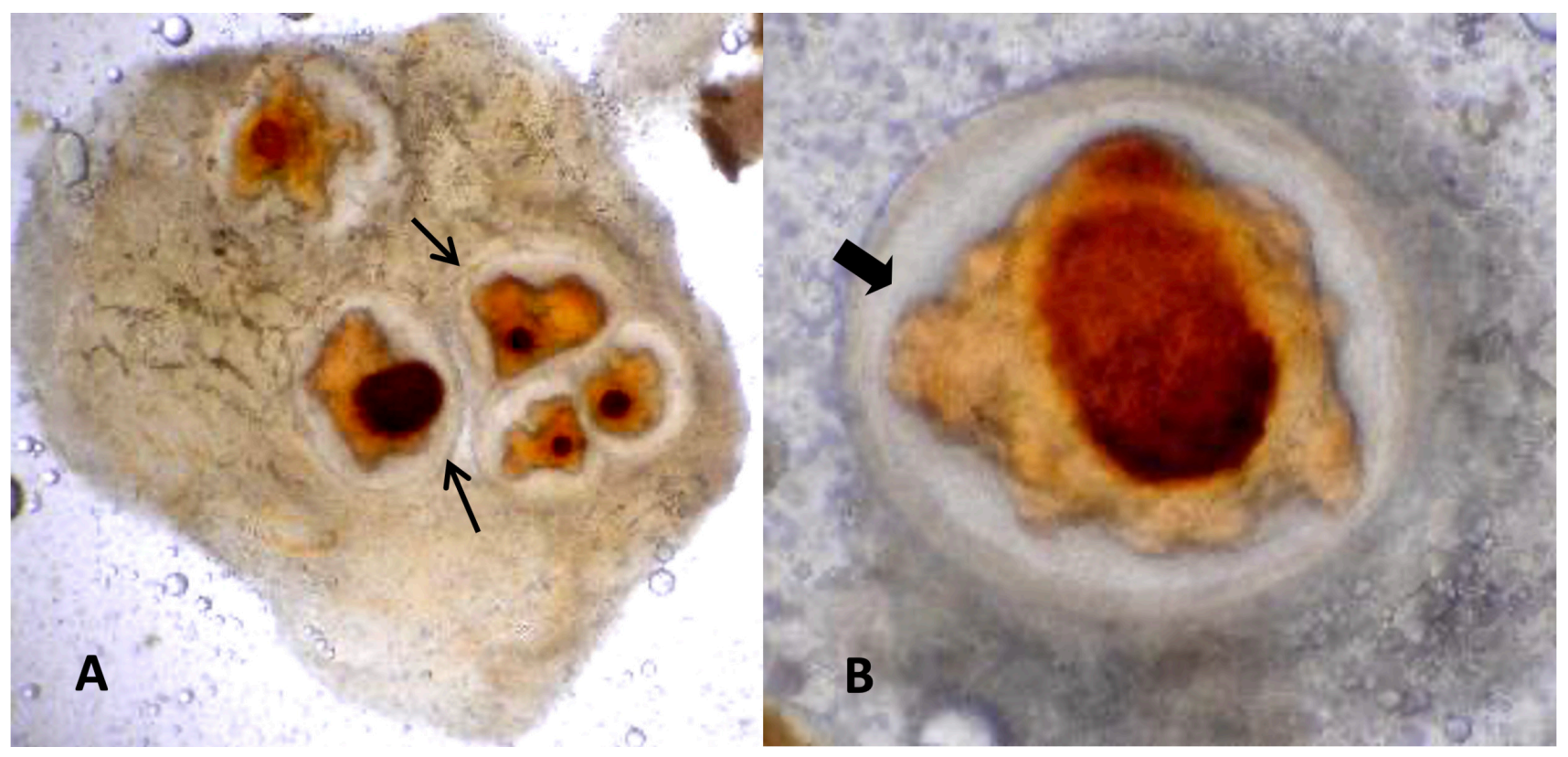

Figure 3. Peritoneal nodules in the perivisceral adipose tissue under light magnification $x 40(\mathrm{~A})$ and $\mathrm{x} 100(\mathrm{~B})$. The arrows point at the connective tissue layers forming a wall around the vaccine inside the nodules. In this case, a mineral oil adjuvanted divalent vaccine against Vibrio anguillarum serotype $\mathrm{O} 1$ and Photobacterium damselae subsp. piscicida had been injected i.p. about 2800 DD before.

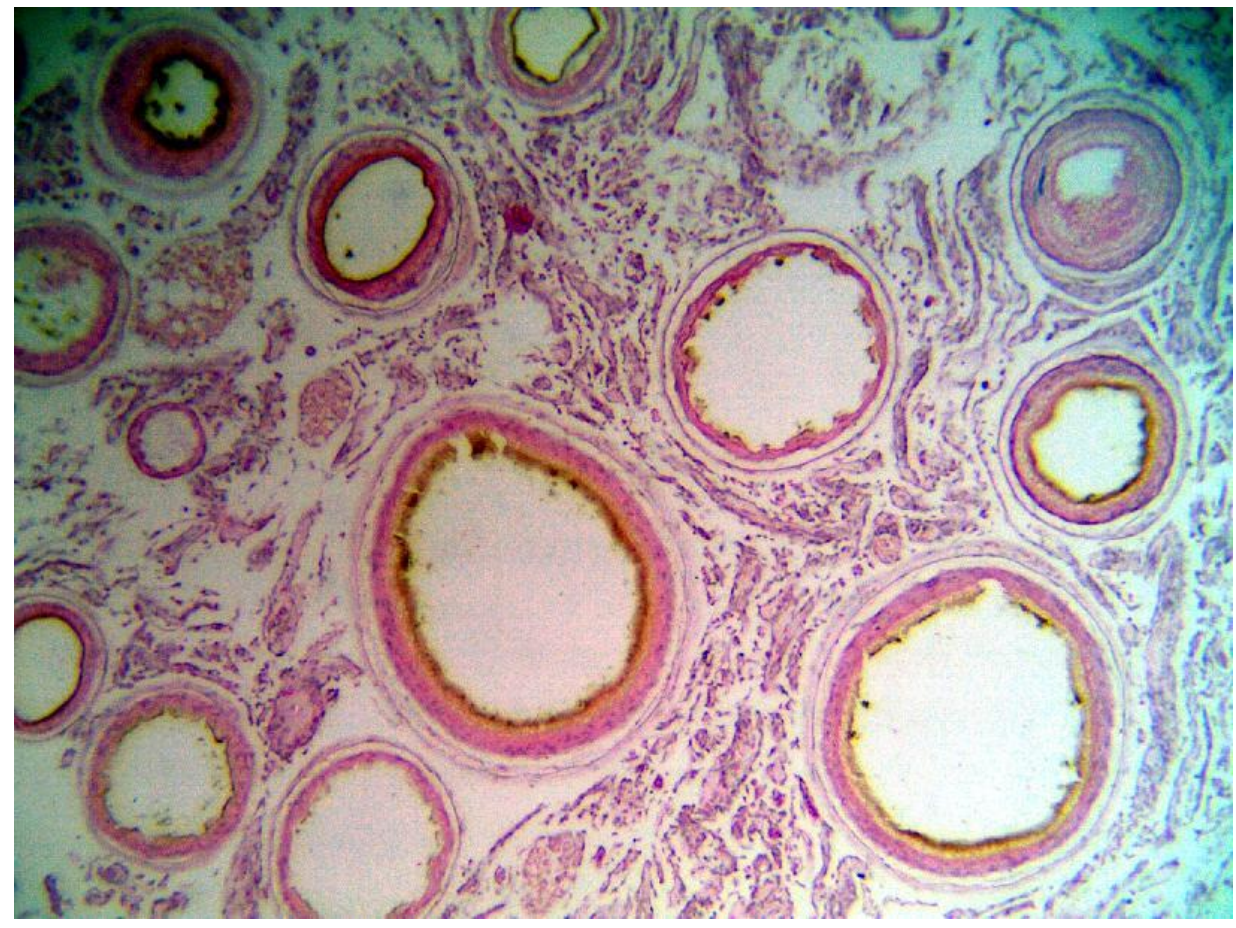

Figure 4. Histology (HE stain, 100X magnification) of peritoneal nodules 2800 DD post i.p. injection with a commercial mineral oil adjuvanted divalent vaccine against Vibrio anguillarum $\mathrm{O} 1$ and Photobacterium damselae subsp. piscicida. The nodule contents, stained brown, comprise the remnants of mineral oil adjuvanted vaccine, which has been mostly removed during the process of fixation and staining. The inner and external layers of connective tissue forming the nodules differ in thickness, presumably in part depending on the section cut.

days post-injection. Adjuvanted vaccines incorporating non-metabolisable and poorly degradable mineral oil (paraffin light liquid) seemed to lead to more intense flogistic effects and to more pronounced adhesions, peritoneal 


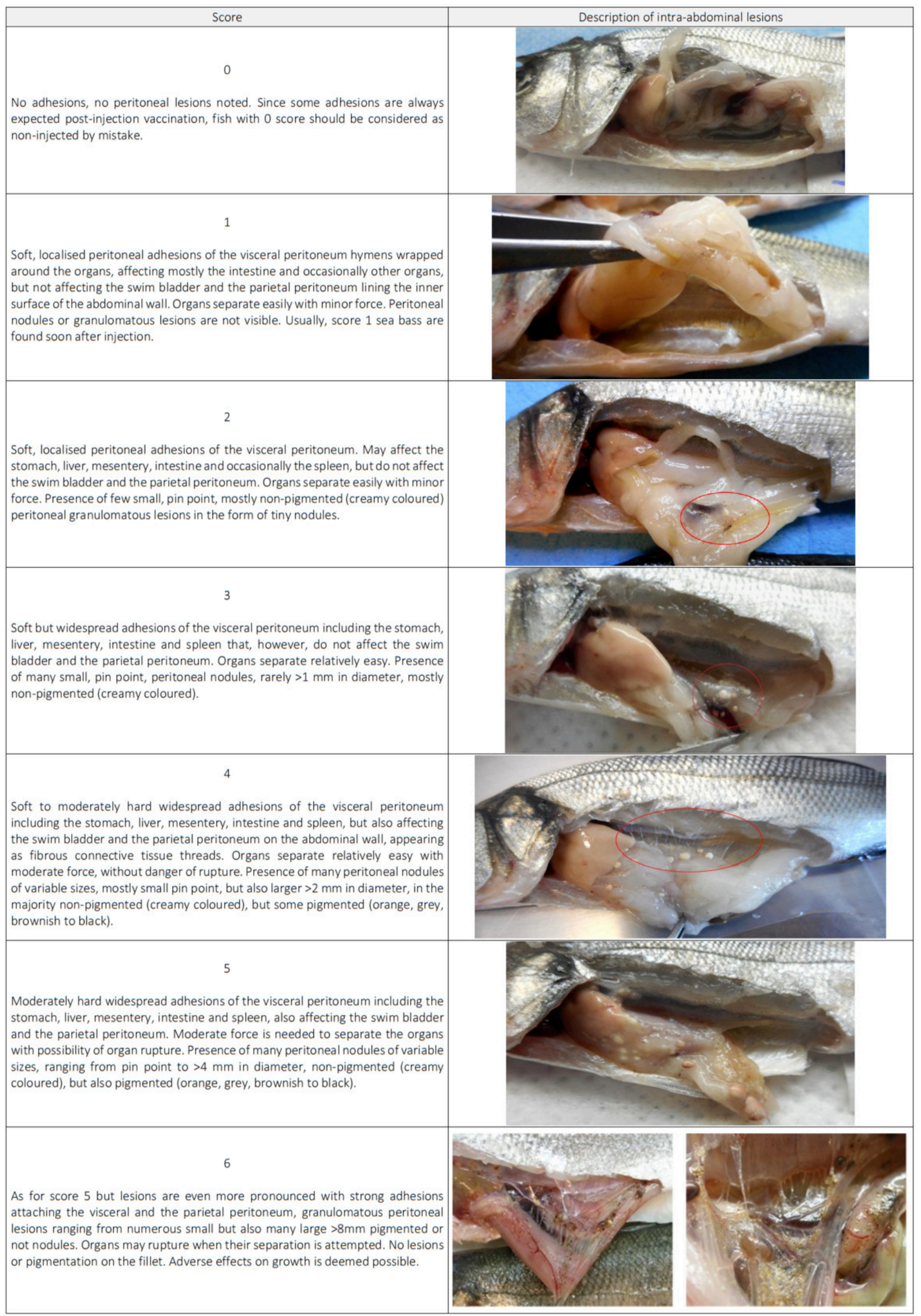

Table 2. Proposed ordinal scale for intra-abdominal side-effects post i.p. vaccination of European sea bass (Dicentrarchus labrax), with description of scoring criteria and illustrative images. 
granuloma formation and pigmentation (orange or brown nodules). Adhesion scores 5 or 6 often resulted after injection with such vaccine formulations. Pigmentation on the internal organs or on the musculature was never observed. Reducing the individual dose by half from $0.1 \mathrm{ml}$ to $0.05 \mathrm{ml}$ by a concomitant increase of the antigen content in the oil-adjuvanted formulations may help diminish the side-effects, notably in fish that are vaccinated at relatively smaller sizes.

Side-effects seemed to peak 1-3 months, or about 650-2000 DD, post-injection. When metabolisable or biodegradable oils were used as vaccine adjuvants, adhesions and granulomatous lesions apparently started to resolve about 3000-3500 DD, or about 6-7 months post-injection. Unfortunately, the current study did not incorporate repeated samplings from the same populations to document the progress of the lesions. There is a need to comparatively evaluate the post i.p. vaccination side-effects at harvest, that is, 18 months, or about 9500 DD post-injection between formulations utilising metabolisable or biodegradable oils against those utilising mineral oil.

Based on the findings of this field-based study, a macroscopic side-effect intensity scale for sea bass is proposed. It is intended to be used as a guide for farm staff to evaluate the intensity of these specific post i.p. injection vaccination side-effects. Focused studies comparing the intensity of side-effects between different vaccine formulations and associating their intensity with growth performance and carcass quality of sea bass at harvest are needed.

\section{Acknowledgements}

The authors are grateful to the Greek aquaculture companies and their staff that contributed fish and resources to help with the on-site samplings. The views expressed in this article are those of the authors. We are grateful to Professor Andy Shinn for his comments as well as to an anonymous reviewer for structural suggestions on the manuscript. 


\section{REFERENCES}

Afonso, A., S. Gomes, J. da Silva, F. Marques, and M. Henrique. 2005. "Side Effects in Sea Bass (Dicentrarchus Labrax L.) Due to Intraperitoneal Vaccination against Vibriosis and Pasteurellosis.” Fish E'Shellfish Immunology 19 (1): 1-16. https://doi.org/10.1016/j.fsi.2004.09.001.

Berg, A, OM Rødseth, A Tangerås, and T Hansen. 2006. "Time of Vaccination Influences Development of Adhesions, Growth and Spinal Deformities in Atlantic Salmon Salmo Salar.” Diseases of Aquatic Organisms 69 (April): 239-48. https://doi.org/10.3354/dao069239.

European Medicines Agency, Committee for Medicinal Products for Veterinary Use, Immunologicals Working Party, 3145550/2010 Consultation. 2011. "European Medicines Agency, Guideline on the Design of Studies to Evaluate the Safety and Efficacy of Fish Vaccines."

Maira, C., Y. Lystad, and S. Alexandersen. 2007. "Safety and Efficacy Results after Vaccination of Cod (Gadus Morhua) with Alpha Marine Vibject.” Pharmaq R\&D study. https://www.pharmaq.no/ sfiles/93/8/file/satstorsk_2007-02.pdf.

Midtlyng, P.J., L.J. Reitan, and L. Speilberg. 1996. "Experimental Studies on the Efficacy and SideEffects of Intraperitoneal Vaccination of Atlantic Salmon (Salmo SalarL.) against Furunculosis.” Fish E'Shellfish Immunology 6 (5): 335-50. https://doi.org/10.1006/fsim.1996.0034.

Muniesa, Ana, Bernardo Basurco, Cristóbal Aguilera, Dolors Furones, Carmen Reverté, Anna Sanjuan-Vilaplana, Mona Dverdal Jansen, Edgar Brun, and Saraya Tavornpanich. 2020. "Mapping the Knowledge of the Main Diseases Affecting Sea Bass and Sea Bream in Mediterranean." Transboundary and Emerging Diseases 67 (3): 1-12. https://doi.org/10.1111/tbed.13482.

Mutoloki, Stephen, Ola B. Reite, Bjørn Brudeseth, Aage Tverdal, and Øystein Evensen. 2006. “A Comparative Immunopathological Study of Injection Site Reactions in Salmonids Following Intraperitoneal Injection with Oil-Adjuvanted Vaccines.”Vaccine 24 (5): 578-88. https://doi.org/ 10.1016/j.vaccine.2005.08.070.

Tafalla, Carolina, Jarl Bøgwald, and Roy A. Dalmo. 2013. "Adjuvants and Immunostimulants in Fish Vaccines: Current Knowledge and Future Perspectives.” Fish E? Shellfish Immunology 35 (6): 1740-50. https://doi.org/10.1016/j.fsi.2013.02.029.

Vendramin, N., S. Zrncic, F. Padros, D. Oraic, A. Le Breton, Zarza C, and N.J. Olesen. 2016. "Fish Health in Mediterranean Aquaculture, Past Mistakes and Future Challenges." Bulletin of the European Association of Fish Pathologists 36 (1): 38-44.

Villumsen, Kasper Rømer, Erling Olaf Koppang, and Martin Kristian Raida. 2015. "Adverse and Long-Term Protective Effects Following Oil-Adjuvanted Vaccination against Aeromonas Salmonicida in Rainbow Trout." Fish छ'Shellfish Immunology 42 (1): 193-203. https://doi.org/ $\underline{10.1016 / j . f s i .2014 .09 .024 .}$. 